\title{
TAGUNG
}

\section{Spendenverhalten zwischen Altruismus und Nutzenmaximierung}

\author{
Frédéric Krumbein*
}

Während der wirtschaftswissenschaftlichen Konferenz ,The Economics of Charitable Giving“ präsentierten Wissenschaftler aus verschiedenen Ländern ihre Erkenntnisse zum Spendenverhalten von Bürgern und Unternehmen.

\section{Steuerliche Anreize für Spenden}

René Bekkers und Elly Mariani sprachen in ihrem Vortrag über die steuerliche Absetzbarkeit von Spenden in den Niederlanden. Spenden an gemeinnützige Organisationen könnten ab 60 Euro oder 1 Prozent des Einkommens abgesetzt werden. Der Maximalbetrag betrage 10 Prozent des besteuerten Einkommens. Bekkers und Mariani analysierten das Spendenverhalten von 0,6 Prozent der niederländischen Steuerzahler. 80-90 Prozent der Steuerzahler spendeten Geld, aber weniger als 5 Prozent der Steuerzahler nähmen die Möglichkeit der Anrechnung ihrer Spenden auf die zu zahlende Steuer in Anspruch. In einer Stichprobe von fast zweitausend Steuerzahlern fragten die Autoren der Studie, warum diese die Abschreibung nicht nutzen. 4,3 Prozent antworteten, sie wüssten nichts davon, 10,9 Prozent füllten generell keine Steuerformulare aus, 28,6 Prozent bräuchten die Steuerabschreibung nicht und 56,5 Prozent antworteten, dass sie die notwendigen Kriterien für die Abschreibung nicht besäßen. Die letzte Antwort sei inkorrekt und basiere auf einem ungenügenden Wissen der Steuerzahler. Die Haushalte erfüllten meistens die Kriterien für eine steuerliche Abschreibung ihrer Spenden. Das Zehntel der Steuerzahler mit den höchsten Einkommen nutze am stärksten

\section{The Economics of Charitable Giving}

Tagung des Arbeitskreises Europäische Integration e.V., des Zentrums für Europäische Wirtschaftsforschung und des Karlsruher Instituts für Technologie

Mit Unterstützung der Europäischen Kommission Mannheim, 8./9. Oktober 2009

Contributed Session 1: Tax Incentives for Charitable Contributions

Is the Charitable Deduction in the Netherlands Treasury Efficient?

Prof. Dr. René BEKKERS, VU University Amsterdam

Elly MARIANI, VU University Amsterdam

Is Charity a Homogenous Good? Variations in the Determinants and Motivations of Charitable Contributions Across Causes

Peter BACKUS, University of Warwick

Government Incentives for Charity Donations and the General Tax Reform

Dr. André PRESSE, Karlsruhe Institute of Technology

Invited Session 1: Tax Incentives for Donations in Germany: An Empirical Analysis of a Reform Proposal

Prof. Dr. Ludwig VON AUER, University of Trier

Invited Session 2: Corporate Social Responsibility and Corporate Philanthropy: Line Up or Trade Off?

Prof. Dr. Theo SCHUYT, VU University Amsterdam

Contributed Session 2: Motivations Behind Volunteering and Giving

Why Without Pay? The Intrinsic Motivation Between Investment and Consumption in Unpaid Labour Supply

Dr. Damiano FIORILLO, University of Naples „Parthenope“

Christmas in Berlin - An Empirical Investigation of Who Donates to the Private Provision of a Public Good and What Drives the Size of Donations

Dr. Beate JOCHIMSEN, Free University Berlin

Category Reporting in Charitable Giving: An Experimental Analysis

Jingping LI, National University of Singapore

* Frédéric Krumbein, Geschäftsführer des Arbeitskreises Europäische Integration e.V., Berlin. 
die Abschreibungsmöglichkeiten. Die anderen neun Zehntel nutzten diese Möglichkeit deutlich weniger. Das unterste Zehntel der Einkommen spende proportional am meisten von ihrem Einkommen.

Peter Backus referierte über die steuerliche Effizienz von Spenden. Eine gängige Grundannahme sei, dass Spenden an gemeinnützige Organisationen für die Gesellschaft und den Staat immer gleich effizient seien. Das bedeute, es spiele aus Sicht des Staates und der Gesellschaft keine Rolle, ob an eine religiöse Organisation oder an eine Einrichtung zur Affenpflege gespendet werde. Diese Grundannahme stimme nach Backus nicht. Backus untersuchte seit 1968 achttausend Haushalte in einer Langzeitstudie in den USA. Die Studie kommt zu dem Ergebnis, dass Spenden für religiöse Zwecke nicht steuereffizient seien. Spenden für säkulare Zwecke seien deutlich steuereffizienter. Es sollte entsprechend keine steuerliche Abschreibung für religiöse $\mathrm{Zwecke}$ erlaubt sein.

André Presse sprach über ein aus seiner Sicht für gemeinnützige Einrichtungen und Unternehmertum sinnvolleres Steuersystem. Steuern sollten erstens transparenter werden. Bei jedem Gut solle dargestellt werden, welcher Anteil an dem Gut eine Steuer sei. Zweitens solle die Mehrwertsteuer, die als Steuer direkt das Gut besteuere, erhöht und als zentrale Steuer eingeführt werden. Die Mehrwertsteuer generiere in Deutschland aktuell 180 Milliarden Euro Einnahmen jährlich. Die Lohnsteuer 140 Milliarden Euro. Die Lohnsteuer solle zugunsten der Mehrwertsteuer reduziert werden. Die Mehrwertsteuer stelle eine geringere Hürde für gemeinnütziges Engagement und Unternehmertum dar, weil sie auch Produktivität besteuere, die nicht aus Arbeitsleistung resultiere. Drittens schlug Presse die Einführung einer negativen Einkommenssteuer für Geringverdiener vor.

Jede Reform des Spendenwesens in Deutschland und anderen Staaten müsse nach Ansicht von Ludwig von Auer mindestens budgetneu-
Invited Session 3: Charitable Memberships, Volunteering, and Discounts: Evidence from a Large-Scale Online Field Experiment

Prof. Dr. Andreas LANGE, University of Maryland

Contributed Session 3: Fund-Raising

Charitable Fund-Raising - Matching Grants or Seed Money: An Application to Employee Matching Grant Schemes

Prof. Bruce D. GRUNDY, Ph.D., University of Melbourne

An Equilibrium Model of Charitable Solicitation, with an Application to Panhandling

Prof. Al SLIVINSKI, Ph.D., University of Western Ontario

Choosing Your Object of Benevolence - A Field Experiment

Bodo ARETZ, Centre for European Economic Research, Mannheim

Keynote Speech: Foundations in Times of Crisis Manfred LAUTENSCHLÄGER, Manfred Lautenschläger Foundation, Heidelberg

Invited Session 4: Inheritance and Charitable Donations

Prof. Richard STEINBERG, Ph.D., Indiana University-Purdue University, Indianapolis

Contributed Session 4: Issues in Charitable Giving

Empirical Evidence on the Relevant Factors Determining Corporate Giving in Portugal

Dr. Daniel TABORDA, Polytechnic Institute of Coimbra

The Last Refuge of a Scoundrel? Patriotism and Tax Compliance

Salmai QARI, Social Science Research Center Berlin

tral sein, da Deutschland und die anderen Industriestaaten in den nächsten Jahren voraussichtlich große Budgetdefizite haben werden. In Deutschland könnten Spenden von der Steuer abgesetzt werden. So seien im Jahr 1998 3,6 Milliarden Euro abgesetzt worden. In anderen europäischen Staaten existierten ebenfalls Systeme der steuerlichen Absetzung von Spenden. Ihnen sei allen gemein, dass sie Anreize für Spenden böten. Spender könnten einen Teil ihrer Kosten, verursacht durch Spenden, absetzen. Der Staat fördere Spenden, weil ein öffentliches Gut teilweise durch Spender finanziert und nicht mehr von der Regierung getragen werden müsse. Die bestehende Spendenpraxis sollte geändert werden. Dabei müssten drei Faktoren berücksichtigt werden: Erstens, es sollten keine zusätzlichen 
Kosten für den Staat entstehen; zweitens, die Spendenpraxis sollte transparenter werden; und drittens, die Kosten zu spenden sollten für alle Haushalte identisch sein. Bislang lohnt sich das Spenden für reiche mehr als für arme Haushalte.

Corporate Social Responsibility in den Niederlanden

Theo Schuyt sprach über ,Corporate Social Responsibility ‘CSR) und die Spendenpraxis von Unternehmen in den Niederlanden. In den Niederlanden spendeten im Jahr 2007 Unternehmen 1,353 Milliarden Euro und damit den zweithöchsten Betrag am gesamten Spendenvolumen nach den privaten Haushalten (1,945 Milliarden Euro). Unternehmen widmeten 35 Prozent ihrer Spenden dem Sport, wie der Unterstützung lokaler Sportvereine, 15 Prozent kulturellen Zwecken und 14 Prozent der Bildung. ,Corporate Giving", das heißt Unternehmensspenden, seien ein Teil der CSR, des ethischen Verhaltens von Unternehmen. CSR könne als eine Pyramide betrachtet werden, bei der die Basis das wirtschaftliche Handeln des Unternehmens darstelle. Darauf aufbauen würden Recht, Ethik und schließlich die Philanthropie., Corporate Giving ' befände sich als Teil der Philanthropie am oberen Ende der Pyramide mit einem relativ geringen finanziellen Volumen. Eine weitere Dimension von CSR neben, Corporate Giving' sei beispielsweise ökologisches Verhalten wie energieeffizientes Wirtschaften, die Reduzierung der Geschäftsreisen oder die Reduzierung des Abfalls. CSR sei ein bekanntes Konzept in Unternehmen. In einer Umfrage unter 654 Unternehmen kannten 59 Prozent aller Unternehmen den Begriff CSR und 52 Prozent beteiligten sich an CSR. 15 Prozent der Unternehmen verfügten über eine eigene CSR-Politik. Nur 15 Prozent der Unternehmen gäben ihren Angestellten die Möglichkeit ehrenamtlich zu arbeiten. 364 der befragten Unternehmen träten als Sponsoren auf oder/und spendeten.
Beweggründe für Geldspenden und ehrenamtliches Engagement

Damiano Fiorillo berichtete über die Motivationen für ehrenamtliches Engagement, das heißt das Spenden von Zeit, in Italien. In einer Studie wurden 2.669 Personen nach ihren Gründen für ehrenamtliches Engagement befragt. Die intrinsische Motivation sei der entscheidende Grund für ehrenamtliches Engagement. Weitere Ergebnisse der Studie seien, dass Menschen mit kleinen Kindern oder Jugendlichen sich signifikant weniger ehrenamtlich engagierten. Außerdem gäbe es einen negativen Zusammenhang mit dem Lebensalter. Ältere Menschen übten seltener ein Ehrenamt aus. Für weitere Forschungen empfahl Fiorillo Analysen über den Zusammenhang zwischen ehrenamtlichem Engagement und dem Grad der persönlichen Zufriedenheit.

Die alljährliche Weihnachtsbeleuchtung des Kurfürstendamms in Berlin durch angrenzende Geschäfte diente Beate Jochimsen als Untersuchungsgegenstand, um die Bereitstellung eines öffentlichen Gutes durch private Institutionen zu analysieren. Bislang existiere wenig Forschung zu Beispielen der Bereitstellung von öffentlichen Gütern. Das Jahr der Untersuchung war 2003. Ein gemeinnütziger Verein, „AG City“, frage jedes Jahr die Anrainerläden, ob sie Geld für die Beleuchtung spenden würden. 2003 seien 1.288 Geschäfte gefragt worden. 81 Geschäfte spendeten Geld. Dabei seien die Namen der Spender und die Branche bekannt, aber nicht die Höhe der Spende. Ziel der Untersuchung sei es, die Motivationen für die erfolgten Spenden herauszufinden. Die Hypothesen von Jochimsen waren folgende: Erstens, Spender möchten als solche veröffentlicht werden; zweitens, die Bereitschaft zum Spenden ist größer am östlichen Ende des Kurfürstendamms, weil die Zahl der Passanten sich von Ost nach West reduziere und die Anrainer uneinheitlich von der Beleuchtung profitierten; drittens, wenig spezialisierte Geschäfte, wie Kaufhäuser, hätten ein größeres Interesse an der Beleuchtung als spezialisierte Geschäfte; und viertens, grö- 
Bere Geschäfte mit einem längeren Schaufenster zum Kurfürstendamm hätten ebenfalls ein größeres Interesse an der Beleuchtung. Die Ergebnisse bestätigten die Hypothesen: Zwischen dem Ort des Geschäfts (Lage im Osten des Kurfürstendamms), der Länge des Schaufensters und der Art des Geschäfts (nicht spezialisiert) sowie dem Spendenverhalten bestehe eine positive Korrelation.

Jingping Li untersuchte die Gründe des Spendens für gemeinnützige Zwecke. Spender seien in erster Linie durch persönliche Vorteile motiviert. In Experimenten an der Fudan Universität in Shanghai, China, seien die Spender, je nach Höhe ihrer Spende, in unterschiedliche Kategorien von Spendern eingeteilt worden. Die Namen der Spender in den jeweiligen Kategorien seien veröffentlicht worden, aber nicht die Höhe ihrer Spende. Spender in höheren Kategorien hätten einen besonderen Status zugewiesen bekommen (wie ,Premiumspender'). Dabei zeige sich, dass die Spender oft exakt den Betrag spendeten, der notwendig sei, um in eine höhere $\mathrm{Ka}-$ tegorie aufzusteigen. Spender seien entsprechend durch das soziale Prestige ihrer Spende motiviert.

Andreas Lange untersuchte die Effektivität unterschiedlicher Formen der Spendenakquise durch gemeinnützige Organisationen. Dabei kam er zu dem Ergebnis, dass gängige Marketingtechniken, wie Attraktivität der Verkäufer oder Rabattaktionen, auch im gemeinnützigen Bereich funktionieren. So verkauften attraktive Frauen mehr Lose von gemeinnützigen Lotterien und sammelten entsprechend mehr Geld ein als andere Spendensammler. Allerdings seien die Spender, die an attraktive Frauen Geld gäben, weniger loyal gegenüber dem gemeinnützigen Zweck der Lotterie. Die Spende sei meist einmalig. Ansonsten determiniere das frühere Spendenverhalten oft das künftige. Menschen, die bereits bei einer ersten Sammelaktion Geld gäben, spendeten wahrscheinlich auch bei einer erneuten Sammlung wieder. Das Internet sei ein weiteres mögliches Instrument zur Spendenakquise. Hier könne das Instrument des Rabatts genutzt werden. Bei einer Sammlung über das Internet für eine linksgerichtete gemeinnützige Organisation sei das Spendenverhalten der 702.890 Spender getestet worden. Der Beitrag für eine Mitgliedschaft in der gemeinnützigen Organisation hätte zwischen 35 US-Dollar, 25 US-Dollar und 25 US-Dollar, ausgewiesen als Rabatt, variiert. Die meisten Spenden seien bei dem rabattierten Mitgliedsbeitrag von 25 US-Dollar eingegangen. Menschen, die früher kein Geld gespendet hätten, seien ebenfalls eher bereit zu spenden, wenn der Mitgliedsbeitrag als Rabatt gekennzeichnet sei. Die Rabattmethode funktioniere also auch im Bereich der gemeinnützigen Organisationen.

\section{Fundraising}

Bruce Grundy referierte über ,Matching ' als Fundraisingstrategie. Diese Methode, bei der ein Dritter zu jeder erhaltenen Spende Geld dazu gebe, funktioniere gut. Gerade Spender von geringen Beträgen spenden mehr, wenn ihre Spende in einem ,Matching '-System aufgestockt werde. Wenn sich der, Matching'Anteil erhöhe, erhöhe sich auch die Spende.

Eine gängige Methode für das Einsammeln von Spenden sei das Verschicken von Briefen mit der Bitte um finanzielle Zuwendungen. Im Allgemeinen seien Menschen eher bereit Geld zu spenden, wenn dieses Geld für eine klar identifizierbare Person aufgewendet werde. Dieser Effekt habe sich in der Empirie bestätigt. Dieser Effekt beschränke sich aber meist auf eine einzelne Person (Opfer mit Namen). Die Übertragbarkeit dieses Effekts auf Spendenbriefe wurde von Bodo Aretz in einem Experiment mit der Organisation „Ärzte für die Dritte Welt“ untersucht. „Ärzte für die Dritte Welt" ist eine humanitäre Organisation, die in fünf Ländern Basisgesundheitsprojekte durchführt. In dem Experiment seien zwei Briefe an potenzielle Förderer versandt worden. Der eine Brief sei ein Standardbrief mit einem allgemeinen Unterstützungsaufruf für 
„Ärzte für die Dritte Welt“ gewesen. In dem anderen Brief konnten die Spender einzelne Länder auswählen, für die ihre Spenden verwendet werden sollten. Es seien über 57.000 Briefe verschickt worden (überwiegend an Ärzte sowie deren Freunde und Angehörige). Die Antwortrate habe circa 11 Prozent betragen und sei damit relativ hoch gewesen. Insgesamt seien etwa 900.000 Euro als Spenden gesammelt worden. Personen, die einzelne Länder auswählen konnten, spendeten signifikant mehr Geld (+18 Prozent). Insgesamt hätten aber nur 3,4 Prozent der Spender diese Möglichkeit wahrgenommen und einzelne Länder ausgewählt. Im Ergebnis habe die Identifizierung mit einem einzelnen Land das Spendenaufkommen erhöht. Allerdings hätten die potenziellen Spender von der Möglichkeit der Identifizierung eines Landes kaum Gebrauch gemacht.

Ehrenamtliches Engagement in Deutschland am Beispiel der „Manfred-LautenschlägerStiftung “

Manfred Lautenschläger sprach über ehrenamtliches Engagement in Deutschland allgemein und im Speziellen von seiner „ManfredLautenschläger-Stiftung“. Lautenschläger ist einer von mehr als 23 Millionen Menschen in Deutschland, die aus unterschiedlichen Beweggründen ehrenamtlich engagiert sind. Soziale Entrepreneure könnten notwendige Veränderungen anstoßen und unterstützen. Die Grundidee sei, die Welt zum Besseren zu verändern. Die ,Manfred-Lautenschläger-Stiftung " unterstütze eine Reihe von verschiedenen Initiativen. So hätte Lautenschläger den Bau eines Kinderkrankenhauses in Heidelberg gefördert. Entscheidend für das Engagement war das Vertrauen in das Management des Krankenhauses. Persönliche Kontakte und Vertrauen in die geförderten Personen und Projekte seien zentrale Kriterien für Förderentscheidungen. Manfred Lautenschlägers persönliche Motivation für sein ehrenamtliches Engagement sei Dankbarkeit für seine Alma Mater, die Universität Heidelberg, und die Stadt Heidelberg für ein erfolgreiches Le- ben. Die Stiftung sei deshalb regional orientiert und nicht national oder global. Ein wichtiger Vorteil dieser regionalen Ausrichtung bestehe in der besseren Überprüfbarkeit der geförderten Projekte.

Bezüglich der staatlichen Förderung innovativer Projekte in Deutschland erwarte Lautenschläger in den nächsten Jahren einen deutlichen Rückgang. Die Finanzkrise und die daraus resultierenden Schulden werden den finanziellen Spielraum des Staates einschränken. Gemeinnützige Initiativen werden teilweise diese Lücke füllen müssen. Unternehmen müssten ebenfalls mehr soziale Verantwortung übernehmen. Die Wissenschaft trage durch Forschung und Beratung dazu bei, die Professionalisierung von gemeinnützigen Einrichtungen weiter zu verbessern. Kritische und selbstkritische Evaluationen von gemeinnützigen Projekten seien entscheidend für eine nachhaltige Wirkung. Gemeinnützige Akteure müssten stärker als bisher aus Fehlern lernen. Viele Formen der Spendenpraxis in Deutschland seien nach wie vor unterentwickelt. Hier könne die Forschung einen wichtigen Beitrag leisten, innovative Ideen zur Verbesserung gemeinnütziger Projekte voranzutreiben. Abschließend plädierte Lautenschläger für die Schaffung einer ,Kultur der Gemeinnützigkeit‘. Der Staat könne viele Probleme alleine nicht lösen. Im Gegensatz zur Nachkriegsgeneration, die in erster Linie viel Geld verdienen wollte, handelten die späteren Generationen solidarischer, frei nach dem Motto von Andrew Carnegie: Es ist eine Schande, als reicher Mann zu sterben.

\section{Verschiedene Erklärungen für Spendenver- halten}

Richard Steinberg sprach über Erbschaften in den USA und wie diese das Spendenverhalten beeinflussen. In den Vereinigten Staaten hätten alte Menschen Milliarden von Dollar an Vermögen angehäuft, die an die nächste Generation weitergegeben würden. Die Frage sei, wie viel aus welchem Grund an karitative 
und soziale Zwecke vererbt werde? Die Religiösität der Menschen sei ein wichtiger Erklärungsfaktor für das Spendenverhalten bei Erbschaften: Menschen spendeten mehr, wenn sie religiös seien. Die ethnische Zugehörigkeit spiele hingegen keine Rolle für das Spendenverhalten. Viele reiche Menschen bauten in der ersten Hälfte ihres Lebens ihr Vermögen auf und gäben es dann in der zweiten Hälfte ihres Lebens für gute Zwecke aus (Beispiele sind Andrew Carnegie und Bill Gates).

Daniel Taborda hat die Spendenpraxis von Unternehmen in Portugal untersucht. Dabei hat er herausgefunden, dass die Marketingausgaben und der finanzielle Erfolg von Unternehmen positiv mit ihrer Spendenbereitschaft korrelieren: Firmen, die hohe
Ausgaben für Marketing hätten und finanziell erfolgreich seien, spendeten mehr als andere.

Salmai Qari hat untersucht, ob eine patriotische Gesinnung die Steuermoral stärke und kam zu einem positiven Ergebnis: Je höher die patriotische Gesinnung, desto besser die Steuermoral. Die patriotische Einstellung wurde anhand mehrerer Fragen gemessen, zum Beispiel mit der Frage: „Wie stolz sind Sie auf ihr Land?" Der Zusammenhang zwischen Patriotismus und Steuermoral sei besonders ausgeprägt in Zeiten des Krieges. Die Regierung könnte diesen Zusammenhang ausnutzen und Patriotismus unter ihren Bürgern fördern. Qari empfahl dies aber nicht, da dann auch der Nationalismus steigen könnte.

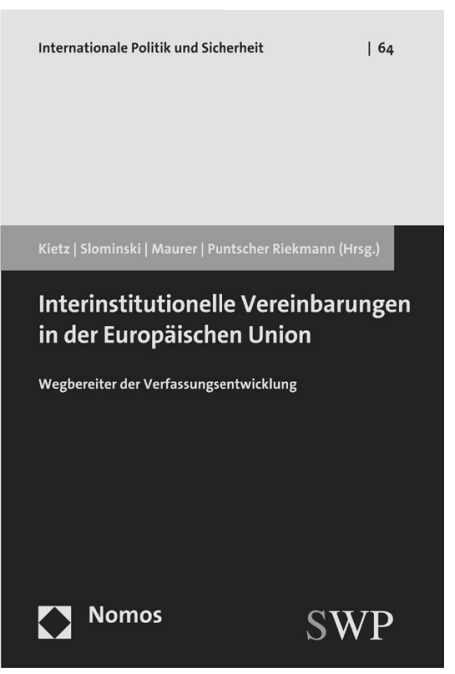

\section{Interinstitutionelle Vereinbarungen in der Europäischen Union}

Wegbereiter der Verfassungsentwicklung Herausgegeben von Daniela Kietz, Dr. Peter Slominski, Dr. Andreas Maurer und Prof. Dr. Sonja Puntscher Riekmann 2010, 340 S., brosch., 49,-€, ISBN 978-3-8329-5373-7 (Internationale Politik und Sicherheit, Bd. 64)

Interinstitutionelle Vereinbarungen dienen der Politikkoordinierung und Konfliktlösung zwischen den EU-Institutionen. Sie ermöglichen ein effizientes Regieren in der EU und wirken als Wegbereiter des institutionellen Wandels. Dieser Band leistet erstmals eine umfassende rechtswie politikwissenschaftliche Einordnung und Analyse der Verhandlungskontexte solcher Vereinbarungen.

Bitte bestellen Sie im Buchhandel oder versandkostenfrei unter - www.nomos-shop.de

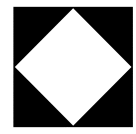

